\title{
Diagnosis of pancreatic lesions using fine needle aspiration cytology: Detection of K-ras point mutations using solid phase minisequencing
}

\author{
J Ihalainen, M Taavitsainen, T Salmivaara, A Palotie
}

\begin{abstract}
Aims-To improve the diagnostic value of fine needle aspiration biopsy of pancreatic lesions using a simple mutation detection method based on the polymerase chain reaction (PCR).

Methods-Fine needle aspirates from 21 suspected pancreatic lesions were analysed for $\mathrm{K}$-ras codon 12 point mutations using solid phase minisequencing. Results-A point mutation in codon 12 of the K-ras gene was detected in 14 of 17 cases of pancreatic carcinoma. No false positive results were recorded. The concordance of the result with routine cytology was $78 \%$. All patients diagnosed as having malignant disease on cytology also had a K-ras point mutation. Additional information on the presence of malignancy was obtained using molecular genetic analysis in two cases.

Conclusions-PCR based minisequencing is a promising method for the analysis of cytological material. K-ras point mutation analysis was modified to enable it to be carried out in a clinical laboratory. Advantages of the method include its simplicity and speed. Adequate sampling guidance is important but analysis can be performed even with small amounts of cellular material.
\end{abstract}

(F Clin Pathol 1994;47:1082-1084)

New technologies for enhancing the diagnostic yield of fine needle aspiration cytology have been reported recently. ${ }^{1}$ Visualisation of tumours using radiological methods and analysis of immunological and genetic markers have improved greatly. The most promising methods for analysing small numbers of cells are those based on the polymerase chain reaction (PCR). Solid phase minisequencing, a single nucleotide primer extension method, has been used in the analysis of both acquired and hereditary point mutations. ${ }^{2}{ }^{3}$ This method is precise, easy to use, and readily automated.

Some malignant disorders have distinctive molecular genetic characteristics. Point mutations of codon 12 of the $\mathrm{K}$-ras gene occur in up to $90 \%$ of pancreatic adenocarcinomas, a feature which may be used in the diagnosis of these cancers. ${ }^{4}$ Alterations in the K-ras gene provide a direct approach to diagnosis and characterisation of a malignancy because their location and nature are well known and well conserved. At present, most clinical laboratory methods used to analyse cancers at the molecular level have been modified from research laboratory protocols. ${ }^{50}$ The purpose of this study was to develop and evaluate simpler methods for performing clinical molecular genetic cytology and to compare its validity with cytological findings. Solid phase minisequencing was chosen because it provides a clear, numerical result and can be easily automated.

\section{Methods}

Biopsy specimens taken from 21 consecutive patients (11 women) with suspected pancreatic cancer were analysed. The mean age of the patients was 64 years (range 32 to 96 years). The patients had been admitted to the Clinic of Radiology at Helsinki University Central Hospital for needle biopsy during 1992 and 1993 because of suspected noninfectious pancreatic lesions. Thereafter, the patients were followed for at least one year. Biopsies were performed using ultrasound guided aspiration with $0.7-0.9 \times 40-150 \mathrm{~mm}$ disposable needles under local anaesthesia. Each needle was attached to a $10 \mathrm{ml}$ syringe in a hand-held biopsy extractor (Cameco, Enebyberg, Sweden). After the samples for routine diagnostic cytology were dispensed, the residual cells were injected into $1 \mathrm{ml}$ physiological saline in a $1.5 \mathrm{ml}$ Eppendorf tube. Routine cytological analysis was performed on alcohol fixed cells stained using the Papanicolau method.

Each study sample was processed for storage within 45 minutes. The cells were centrifuged briefly at $10000 \mathrm{rpm}$ and the supernatant fluid discarded. The cells were stored in $-70^{\circ} \mathrm{C}$. The cell samples were prepared for PCR as described previously. ${ }^{37}$ The primers, one of which was biotinylated at the 5 ' end, used for nested PCR amplification of the region of interest are presented in table 1 . PCR was performed using Taq DNA polymerase and amplification buffer from the same manufacturer (Promega, Madison, Wisconsin, USA). The reaction conditions were essentially as described before. ${ }^{3}$ Techne (Techne, Duxford, Cambridge, UK) or LKB (Pharmacia LKB Biotechnology, Bromma, Sweden) PCR thermocyclers were used. The temperature protocol was $95^{\circ} \mathrm{C}$ for one minute, $55^{\circ} \mathrm{C}$ for one minute, and $72^{\circ} \mathrm{C}$ for one minute.

For minisequencing analysis of the K-ras codon 12, PCR products amplified using 
Table $1 P C R$ and sequencing primers used in $K$-ras codon 12 minisequencing analysis

\begin{tabular}{ll}
\hline Primer & Sequence \\
\hline PCR 5' primer & AGG CCT GCT GAA AAT GAC TG \\
PCR 3' primer & AAG ATT TAC CTC TAT TGT TGG \\
Nested 5' primer & GAC TGA ATA TAA ACT TGT GG \\
Nested 3' primer (biotinylated) & CCT CTA TTG TTG GAT CAT AT \\
Codon 12:1 5' sequencing primer & AAC TTG TGG TAG TTG GAG CT \\
Codon 12:2 5' sequencing primer & ACT TGT GGT AGT TGG AGC TT \\
\hline
\end{tabular}

biotinylated primers were bound to streptavidin coated microtitration plates. The PCR products were then denaturated and the minisequencing reaction performed as described before (figure). ${ }^{8}$ The reaction proceeded for 60 minutes at $37^{\circ} \mathrm{C}$. The unbound reaction components were then washed away using an automatic washer (Delfia Platewash, Wallac, Turku, Finland). The microtitration plates used (ScintiStrips, Wallac) were fabricated from scintillating plastic and only needed to be air dried for 30 minutes before being inserted into a microtitration plate format scintillation counter (Wallac Microbeta, Wallac). In minisequencing analysis point mutations are detected by comparing the incorporated radioactivities (counts per minute) of the normal and aberrant nucleotide (figure), and are expressed as a ratio of incorporation of normal and abnormal nucleotides ( $R$ value). When screening for mutations a mixture containing all the aberrant nucleotides in one reaction was used. The positive findings were confirmed using specific single nucleotide primer extension reactions. The results are presented in table 2 .

Both positive (SW 116 cell line, ATCC, Rockville, Maryland, USA) and negative (DNA from healthy subjects) internal controls were included in each series. A + 3SD increase from the control value was indicative of a positive result. Two parallel minisequenc-

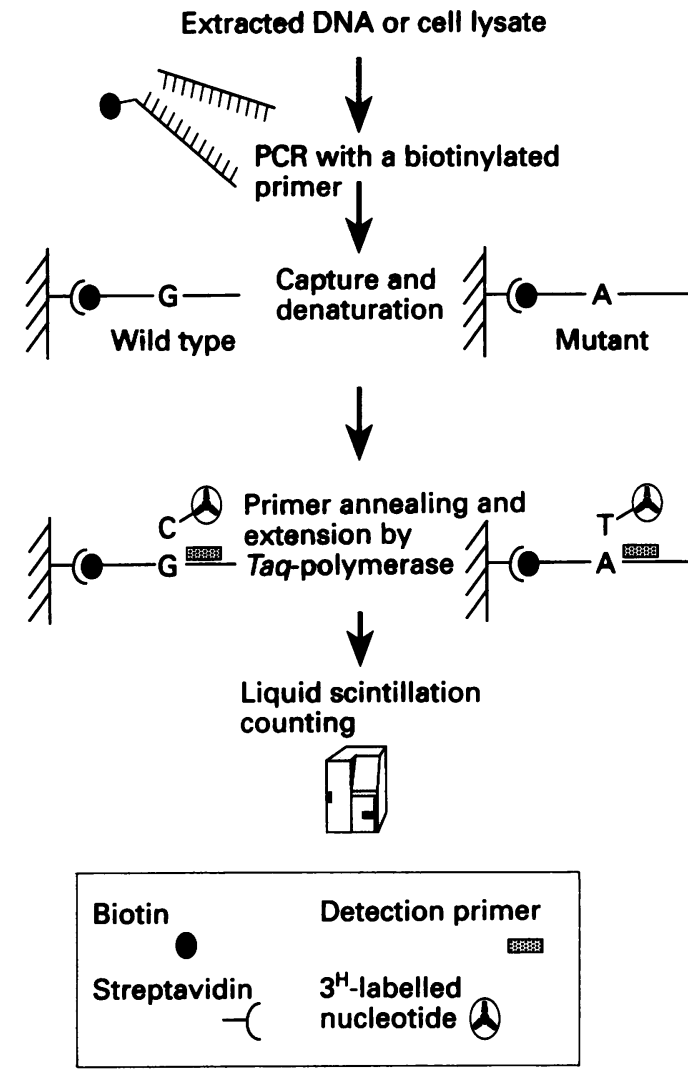

A flow chart of the minisequencing assay. The minisequencing procedure was performed in 96 well microtitration plates. The plates were coated with streptavidin and served as the solid phase in the assay. The single nucleotide primer extension reactions for the normal and mutant nucleotides were performed in separate wells. The amount of mutated compared with normal PCR product was indicated by the incorporated radioactivity in the corresponding wells of the microtitration plate. The ratio of these radioactivities was defined as the $R$ value. For example, if the sample only contains normal DNA the activity in the $G$ reaction well is about 3000 counts per minute. The corresponding activity in the $A, C$, and $T$ reactions is essentially background - for example, 50 counts per minute. The abnormal count rate was divided by the normal one. Thus, the $R$ value of the example is $0 \cdot 17$.

ing reactions were performed for each nucleotide and sample, and served as additional controls for potential technical faults.

pancreatic lesions

\begin{tabular}{|c|c|c|c|c|}
\hline \multirow{2}{*}{$\begin{array}{l}\text { Case } \\
\text { No }\end{array}$} & \multirow[b]{2}{*}{ Cytological diagnosis } & \multirow[b]{2}{*}{ Final diagnosis } & \multicolumn{2}{|c|}{ Minisequencing results } \\
\hline & & & $R$ values $* 12: 1$ & $R$ value $12: 2$ \\
\hline 1 & Cyst & Pancreatic cancer & 0.059 & 0.086 \\
\hline 2 & Inflammation & Pancreatic pseudocyst & 0.049 & 0.059 \\
\hline 3 & Inflammation & Pancreatic cancer & 0.093 & 0.078 \\
\hline 4 & Suspected carcinoma & Pancreatic cancer & 0.077 & $2 \cdot 082$ \\
\hline 5 & Carcinoma & Pancreatic cancer & 0.052 & 0.426 \\
\hline 6 & None & Sclerosing cholangitis & 0.015 & 0.029 \\
\hline 7 & Carcinoma & Pancreatic cancer & 0.159 & 0.034 \\
\hline 8 & Carcinoma & Pancreatic cancer & 0.039 & $3 \cdot 449$ \\
\hline 9 & Normal & Pancreatic cancer & 0.02 & 0.501 \\
\hline 10 & Carcinoma & Pancreatic cancer & 0.039 & $3 \cdot 754$ \\
\hline 11 & Carcinoma & Pancreatic cancer & 0.013 & 0.461 \\
\hline 12 & Carcinoma & Pancreatic cancer & $0 \cdot 31$ & 0.057 \\
\hline 13 & Carcinoma & Pancreatic cancer & 0.026 & $0 \cdot 242$ \\
\hline 14 & Carcinoma & Pancreatic cancer & 0.026 & 1.866 \\
\hline 15 & Carcinoma & Pancreatic cancer & 0.047 & 8.979 \\
\hline 16 & Carcinoma & Pancreatic cancer & 0.026 & 0.295 \\
\hline 17 & None & Benign cystadenoma & 0.022 & 0.035 \\
\hline 18 & Inadequate sample & Pancreatic cancer & 0.021 & 0.033 \\
\hline 19 & Carcinoma & Pancreatic cancer & 0.033 & 0.461 \\
\hline 20 & Carcinoma & Pancreatic cancer & 0.027 & 0.353 \\
\hline \multirow[t]{4}{*}{21} & None & Intestinal lymphoma & 0.028 & 0.039 \\
\hline & & Control mean & 0.039 & 0.045 \\
\hline & & Control SD & 0.02 & 0.02 \\
\hline & & Mean + 3 SD & 0.099 & $0 \cdot 105$ \\
\hline
\end{tabular}

The $R$ values of samples with mutations are in italics and the two borderline values are in bold. The $R$ value was obtained by dividing the reactivity (counts per minute) of mutant nucleotides by The $R$ value was obtained by dividing the reactivity (counts per
the radioactivity (counts per minute) of the normal nucleotide.

the radioactivity (counts per minute) of the normal nucleotide. The $R$ value of the control group was determined by analysing five healthy subjects, one patient
with a lymphoma who was not expected to have a K-ras mutation, and three inflammatory cell with a lymphoma who was not expected to have a K-ras mutation, and three inflammat
samples from bronchoalveolar lavage fluid specimens from patients with lung infections.

\section{Results \\ Minisequencing revealed $\mathrm{K}$-ras codon 12 mutations in 14 of 21 cases. Of these muta- tions, 12 occurred in the second nucleotide of the codon and two at the first. This finding agrees with earlier reports of the distribution of K-ras mutations in pancreatic cancer. ${ }^{4}$ \\ When minisequencing data were compared with the primary cytopathological reports, all 12 patients with pancreatic adenocarcinoma on cytology had point mutations in the K-ras gene. Two other patients, one cytologically normal, the other with suspected pancreatic cancer, also had point mutations. Clinical data obtained at follow up and histological biopsy reports confirmed their final diagnosis} as pancreatic adenocarcinoma. Two of the 21 patients were diagnosed as having pancreatic inflammation and a cyst. A third patient had inadequate sample. They were normal on the point mutation analysis, but later diagnosed 
as having carcinomas by other means (histology and clinical examination). One patient had an intestinal lymphoma diagnosed on cytology and histology. His sample was negative on K-ras point mutation analysis.

Two patients (cases 1 and 3) had borderline $R$ values ( +2 SD above normal). They did not show significant aberrant incorporation on single nucleotide incorporation analysis and thus were not regarded as having point mutations. Both of these patients had pancreatic adenocarcinomas.

\section{Discussion}

We studied the effect of fast and reliable point mutation analysis for the detection of malignancy using fine needle aspiration biopsy specimens of pancreatic lesions. Our results show that the specificity and sensitivity of $\mathrm{K}$-ras point mutation analysis of ultrasound guided pancreatic fine needle biopsy specimens are as good as those of standard microscopic cytology. K-ras point mutations occur in up to $90 \%$ of pancreatic adenocarcinomas. ${ }^{9}$ In this study all carcinomas diagnosed on cytology also had a point mutation.

Samples with substantial amounts of necrosis or inflammatory changes are more likely to give false negative results in PCR based tests. This may be because of a lack of malignant cells, dense inflammatory cell background, or the presence of bacterial DNA digesting enzymes. Our observations confirm that a good sampling technique and proper needle localisation are prerequisites for successful fine needle biopsy molecular cytology. There is a $10-40 \%$ false negative rate in the diagnosis of pancreatic malignancies with guided biopsy. ${ }^{10}$ The pancreatic mass comprises both malignant cells and secondary inflammatory areas which explains the inevitable false negative results.

On the other hand, samples with good morphology and a large number of apparently normal cells are good candidates for amplification using PCR. The efficacy of PCR in the surveillance of residual acute leukaemic disease has been reported before. ${ }^{11}$ In this study the sample morphologically diagnosed as normal pancreatic tissue, but containing malignant cells on minisequencing, provides evidence that PCR amplification is a highly sensitive method for detecting malignant cells against a normal cell background. The two cancer patients with borderline $R$ values show that the sensitivity of this method for detecting mutations can be increased even further. In these cases we could not confirm the pres- ence of a point mutation using specific nucleotide incorporation analysis. This may be because only one nucleotide is present in the specific reaction. In the screening reaction three different nucleotides are available, enabling the polymerase to elongate the chain following mutation. The extra nucleotides potentiate the search for mutations. Thus, the reaction may be more sensitive to very small amounts of mutated alleles if a mixture containing all three possible mutant nucleotides is used. Magnetic microspheres can also be used as a solid phase, increasing the sensitivity of the assay. ${ }^{312}$

The methods used in this study are relatively easy to use and maintain, and can be modified and adapted to non-radioactive detection systems. The protocols can be standardised and the procedure is almost identical for any known point mutation, facilitating the analysis of multiple point mutations in a single minisequencing series.

In conclusion, PCR based analysis of ultrasound guided fine needle biopsy material is a promising method for enhancing diagnostic accuracy in central cytopathology laboratories. The basic principles of diagnostic cytology, however, must also be taken into account.

1 Byley ID. Update on special techniques in routine cytopathology. F Clin Pathol 1993;46:881-5.

2 Syvänen A-C, Aalto-Setälä K, Harju L, Kontula K, Söderlund $\mathrm{H}$. A primer-guided nucleotide incorporation assay in the genotyping of apolipoprotein E. Genomics assay in the get

3 Syvänen A-C, Söderlund H, Laaksonen E, Bengtstöm M, Turunen $M$, Palotie A. N-Ras gene mutations in acute myeloid leukemia: accurate detection by solid-phase minisequencing. Int $\mathcal{f}$ Cancer 1992;50:713-18.

4 Bos JL. Ras oncogenes in human cancer: a review. Cancer Res 1989;49:4682-9.

5 Ridanpää $M$, Husgafvel-Pursiainen $K$. Denaturing gradient gel electrophoresis (DGGE) assay for K-ras and N-ras genes: detection of K-ras point mutations in human lung tumor DNA. Hum Mol Gen 1993;2:639-44.

6 Urban T, Ricci S, Grange J-D, Lacave R, Boudghene F, rban T, Ricci S, Grange J-D, Lacave R, Boudghene F,
Breittmayer F, et al. Detection of c-Ki-ras mutation by Breittmayer $\mathrm{F}$, et al. Detection of c-Ki-ras mutation by
PCR/RFLP analysis and diagnosis of pancreatic adenoPCR/RFLP analysis and diagnosis of pancreatic
carcinomas. $₹$ Natl Cancer Inst 1993;85:2008-12.

7 Syvänen A-C, Ikonen E, Manninen T, Bengtström M, Söderlund $\mathrm{H}$, Aula $\mathrm{P}$, et al. Convenient and quantitative determination of the frequency of a mutant allele using solid-phase minisequencing: application to aspartylglucosaminuria in Finland. Genomics 1992;12:590-5.

8 Ihalainen J, Siitari H, Laine S, Syvänen A-C, Palotie A. Towards automatic detection of point mutations: use of scintillating microtitration plates in solid phase minisescintillating microtitration plates in solid
quencing. Biotechniques 1994;16:938-43.

9 Hruban RH, Van Mansfield ADM, Offerhaus GJA VanWeeringh DHJ, Allison DC, Goodman SN, et al. $\mathrm{K}$-ras oncogene activation in adenocarcinoma of the human pancreas. Am $\mathcal{F}$ Pathol 1993;143:545-54.

10 Mueller PR. Pancreatic biopsy: Striving for excellence. Radiology 1993;187:15-6.

11 Negrin RS, Blume KG. The use of the polymerase chain reaction for the detection of minimal residual malignan disease. Blood 1991;78:255-8.

12 Palotie A, Syvänen A-C. Development of molecular genetic methods for monitoring myeloid malignancies. Scand f Clin Lab Invest 1993;53(Suppl 213):29-38. 